\title{
MODULATIONAL INSTABILITY OF ION-ACOUSTIC
}

\section{TURBULENCE}

F. Yasseen and J. Vaclavik 
Modulational Instability of Ion-Acoustic Turbulence

\author{
F. Yasseen and J. Vaclavik \\ Centre de Recherches en Physique des Plasmas \\ Association Euratom - Confédération Suisse \\ Ecole Polytechnique Fédérale de Lausanne \\ CH-1007 Lausanne / Switzerland
}

\begin{abstract}
The adiabatic interaction of low frequency ion-acousticlike perturbations with high frequency ion acoustic turbulence is studied. The turbulence is found to become unstable provided certain conditions are met. These conditions are explicitly derived.
\end{abstract}




\section{Introduction}

The stability of stationary turbulence is of recent interest in laboratory $^{1}$ and astrophysica ${ }^{2}$ plasmas. To determine whether or not a given type of turbulence is stable, it is sufficient to examine its interaction with a certain type of wave. If no growing mode is excited, the turbulence is stable with respect to that sort of perturbation and is therefore unaffected by noise of the same kind.

Much work has been devoted to the stability of Langmuir turbulence. One knows, for example, that Langmuir turbulence can be modulationally unstable if its spectrum is sufficiently narrow or its level sufficiently high $^{3}$. On the other hand, little is known about the stability of ionacoustic turbulence.

The objective of this paper is to investigate whether high frequency ion acoustic turbulence is unstable with respect to low frequency ionacoustic perturbations, i.e. within the context of the "adiabatic wave interaction with weak turbulence". The arrangement of this paper is as follows : In section II, the master dynamical equations in wavenumber space, which describe the nonlinear interaction of ion acoustic waves, are used to construct a set of self-consistent equations describing the adiabatic interaction of small-amplitude coherent waves with weak turbulence, by means of a method proposed recent $1 y^{4}$. These equations 
are then linearized, in section III, and used to deduce an appropriate dispersion relation. The latter will be analyzed in section IV within the "cold wave" approximation.

II. Basic Equations

Consider a collisionless, non-magnetized plasma composed of a cold ion fluid and a warm isothermal electron fluid. If electron inertia effects are neglected, the plasma will be governed by the equations of momentum transfer

$$
\frac{\partial}{\partial t} \underline{v} \quad=-\underline{\nabla} \phi-(\underline{v} \cdot \underline{\nabla}) \underline{v} .
$$

continuity

$$
\frac{\partial}{\partial t} n=-\underline{\nabla} \underline{v}-\underline{\nabla}(n \underline{v}),
$$

with the Poisson equation

$$
-\nabla^{2} \phi=n+1-\exp (\phi),
$$

for closure, where $\underline{v}$ and $\phi$ are the ion velocity and the electrostatic potential, and $n$ denotes the deviation of the ion density from equilibrium. Here, length, time, electrostatic energy, ion density and ion velocity are measured in units of the Debye length $\lambda_{D}$, ion plasma period $\omega_{p_{i}}^{-1}$, electron thermal energy $k_{B} T_{e}$, equilibrium density $n_{\bullet}$ and ion sound speed $c_{s}$, in that order.

If the electrostatic energy is much smaller than the thermal energy, i.e. if $\phi \ll 1$, we may expand equation (3), retaining only quadratic and 
cubic nonlinearities, which are sufficient for our purpose. We may then cast equations (1) and (2) by standard techniques ${ }^{5}$ into the corresponding master dynamical equations in wavenumber space for the wave variable $C_{p}^{s}$

$$
\begin{aligned}
& \left(i \frac{\partial}{\partial t}-w_{p}^{s}\right) C_{p}^{s}=\sum_{s^{\prime}, s^{\prime \prime}} \sum_{p^{\prime}} V_{p, p^{\prime}, p-p^{\prime}}^{s} C_{p^{\prime}}^{s^{\prime}} C_{p-p^{\prime \prime}}^{s^{\prime \prime}} \\
& +\sum_{s^{\prime}, s^{\prime \prime}, s^{\prime \prime}} \sum_{p^{\prime} p^{\prime \prime}} K_{p_{1} p^{\prime}, p^{\prime \prime}, s^{\prime \prime \prime}-p^{\prime}-p^{\prime \prime} C_{p^{\prime}}^{s^{\prime}} C_{p^{\prime \prime}}^{s^{\prime \prime}} C_{p-p^{\prime}-p^{\prime \prime}}^{s^{\prime \prime}},}
\end{aligned}
$$

where $p^{\prime}$ s are wavevectors, $s$ 's are indices running throught the eigenmodes of the linear system, and $V$ and $K$ are respectively the quadratic and cubic interaction kernels. The normalization of the wave variable is chosen such that to first order, $C_{p}=\phi_{p}$. We find

$$
\begin{aligned}
& V_{p \cdot p^{\prime} \cdot p^{\prime \prime}}^{s s^{\prime}}=\frac{\omega_{p}^{s}}{4 \beta_{p}^{2}}\left[\frac{\left(p_{-}^{\prime} \cdot p_{-}^{\prime \prime}\right)}{\omega_{p^{\prime}}^{s} \omega_{p^{\prime \prime}}^{s^{\prime \prime}}} \beta_{p}^{2}+\frac{\left(\underline{p} \cdot \underline{p}^{\prime}\right)}{\omega_{p}^{s} \omega_{p^{\prime}}^{s} \beta_{p}^{2 \prime}}+\frac{\left(p_{-} \cdot p_{-}^{\prime \prime}\right)}{\omega_{p}^{s} \omega_{p^{\prime \prime}}^{s 1}} \beta_{p^{\prime}}^{2}-1\right], \\
& K_{p \cdot p^{\prime} \cdot p^{\prime \prime} \cdot p^{\prime \prime \prime}}^{s s^{\prime \prime \prime}}=\frac{\omega_{p}^{s}}{12 \beta_{p}^{2}}\left[\frac{1}{\beta_{p-p^{\prime}}^{2}}+\frac{1}{\beta_{p-p^{\prime \prime}}^{2}}+\frac{1}{\beta_{p-p^{\prime \prime \prime}}^{2}}-1\right] \text {, }
\end{aligned}
$$

where

$$
\omega_{p}^{s}=s \frac{p}{\beta_{p}} \Delta g_{n}\left(p_{t}\right), \beta_{p}=\left(1+p^{2}\right)^{1 / 2}, s=+,-.
$$


Notice that

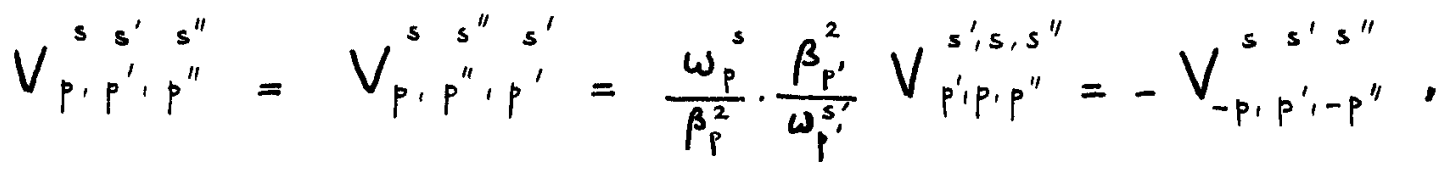

and that $K$ is invariant with respect to all permutations of the primed indices.

We proceed now to construct a set of self-consistent equations describing the interaction of a low-frequency sma11-amplitude coherent wave with high frequency weak turbulence. To do so, we assume that the wave variable $C_{p}^{s}$ consists of a stochastic part, $\tilde{C}_{p}^{s}$, and a coherent one, $\bar{C}_{p}^{s}$, i.e.

$$
C_{p}^{s}=\bar{C}_{p}^{s}+\tilde{C}_{p}^{s}
$$

with $\bar{C}_{p}^{s} \equiv\left\langle C_{p}^{s}\right\rangle,\left\langle\tilde{C}_{p}^{s}\right\rangle \equiv 0$, where \langle\rangle indicates an ensemble average.

By averaging (4), we find to first order in $\bar{C}_{q}$ and $I_{k}, q$

$$
\begin{aligned}
& \left(i \frac{\partial}{\partial t}-\omega_{q}^{s}\right) \bar{C}_{q}^{s}=\sum_{s^{\prime}} \sum_{k} V_{q, q / 2+k, q / 2-k}^{s} s_{k, q}^{\prime} s^{\prime} \\
& +3 \sum_{s^{\prime}, s^{\prime \prime}} \sum_{q^{\prime}, k}^{s^{\prime}} K_{q, q^{\prime}, \frac{q-q^{\prime}}{2}+k, \frac{q^{\prime} q^{\prime}-k}{2}-I_{k, q-q^{\prime}}^{s} \bar{C}_{q^{\prime}}^{s^{\prime \prime}},}^{s^{\prime \prime}}
\end{aligned}
$$

where $I_{k, q}^{s} \cdot \delta_{s, s^{\prime}} \equiv\left\langle C_{q / q}^{s}, \quad C_{q / 2}^{s^{\prime}-k}\right\rangle$ is the correlation function. In a straightforward manner, we construct a similar equation for $I_{k, q}{ }^{3}$, from equation (4), (9) and (10). We find 
- $6-$

$$
\begin{aligned}
& \left(i \frac{\partial}{\partial t}-\omega_{k+q / 2}^{s}-\omega_{-(k-q / 2)}^{s}\right) I_{k, q}^{s}=2 \sum_{s^{\prime}} \sum_{q^{\prime}} \bar{C}_{q^{\prime}}^{s^{\prime}} .
\end{aligned}
$$

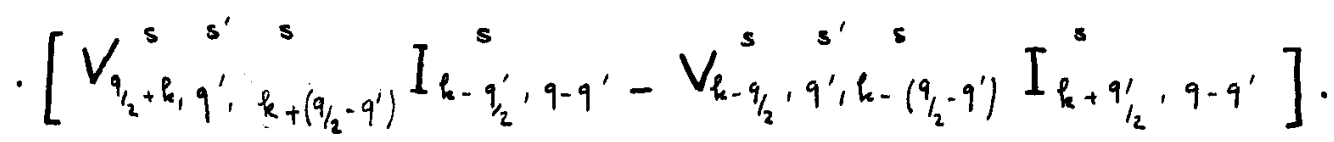

This system of equations $[(10)-(11)]$ is quite complicated. However, it may be considerably simplified if the "adiabatic approximation" is intraduced. ${ }^{6}$ To do so, we assume that the coherent wave and the turbulence are far apart in frequency-wavenumber space, e.g. we consider only low frequency coherent waves and only high frequency turbulence.

In other words

$|q| \ll|k|,\left|\omega_{q}\right| \ll\left|\omega_{k}\right|, \bar{\psi}_{k}=\tilde{\psi}_{q}=0$,

where $q$ refers to the coherent wave and $k$ to the turbulence. This approximation ensures the existence of a small parameter, $|q / k|$ in which (10) - (11) may be expanded. For our purpose, zero order is sufficient for (10). We obtain

$$
\begin{aligned}
& \left(i \frac{\partial}{\partial t}-w_{q}^{s}\right) C_{q}^{s}=\sum_{s^{\prime}} \sum_{k} V_{q, k_{1}-k}^{s s^{\prime} s^{\prime}} I_{k, q}^{s^{\prime}} \\
& +3 \sum_{s^{\prime}, s^{\prime \prime}} \sum_{q^{\prime}, k} K_{q, q^{\prime}, k_{1}-k}^{s^{\prime} s^{\prime} s^{\prime \prime}} \bar{I}_{k, q-q^{\prime}}^{s^{\prime \prime}}, \bar{C}_{q^{\prime}}^{s^{\prime}} .
\end{aligned}
$$


Equation (11), on the other hand, will have to be expanded to first order. We obtain

$$
\begin{aligned}
& \left(i \frac{\partial}{\partial t}-\underline{q} \cdot \frac{d \omega_{k}^{s}}{d \underline{\underline{t}}}\right) I_{k, q}^{s}=-2 \sum_{s^{\prime}} \sum_{q^{\prime}} C_{q^{\prime}}^{s^{\prime}} \cdot\left\{\left(\underline{q^{\prime}} \cdot \frac{\partial}{\partial \underline{\underline{g}}} I_{k, q-q^{\prime}}^{s} V_{k, q^{\prime}, k}^{s s^{\prime} s}-\right.\right. \\
& \left.-\left[\left.\underline{q} \cdot \frac{\partial}{\partial \underline{p}} V_{p, q^{\prime}, k}^{s s^{\prime} s}\right|_{p=k}+\left.\left(\underline{q}-2 \underline{q}^{\prime}\right) \cdot \frac{\partial}{\partial p} V_{k, q^{\prime}, p}^{s s^{\prime} s}\right|_{p=k}\right] I_{k, q-q^{\prime}}^{s}\right\}
\end{aligned}
$$

where $\omega_{-k}^{s}=-\omega_{k}^{s}$ has been used.

III. Dispersion Relation

At this stage, we make two further assumptions. First, that in the absence of the coherent wave, the plasma is in a stationary state where only the turbulent waves with positive wave velocities along the $z$-axis are excited. This situation is often met in experiments ${ }^{7-10}$. Second, that in the presence of the coherent wave, a small, time-dependent change in the level of the turbulence will occur, i.e.

$$
I_{k, q}^{+}(t)=I_{k} \cdot \delta_{q, 0}+I_{k, q}^{1}(t)
$$

where $I^{+}, I$ and $I^{1}$ refer, respectively, to the turbulence with or without the coherent wave, and the small change brought about by the coherent wave, with the condition $\left|I_{k, q}^{1}\right| \ll I_{k}$. 
Introducing (15) into equations (13) and (14), linearizing, and assuming a time dependence of the form $\exp (-i \Omega t)$ for both the coherent wave and the perturbation of the turbulence, we find

$$
\begin{aligned}
& \left(\Omega-\omega_{q}^{s}\right) \bar{C}_{q}^{s}=\sum_{s^{\prime}} \sum_{k} C_{q}^{s^{\prime}}\left[3 K_{q, q^{\prime}, k_{1}-k}^{s s^{\prime}+1+} \frac{\omega_{k}^{+}}{\beta_{k}^{2}} N_{k}-\right. \\
& \left.-2 \frac{\underline{q} \cdot \frac{d N_{k}}{d \underline{\underline{t}}}}{\Omega-q \cdot \frac{d \omega_{k}^{+}}{d \underline{t}}} \cdot \frac{\omega_{k}^{+}}{\beta_{k}^{2}} V_{q, k_{1}-k}^{s++} V_{k, q, k}^{+s^{\prime}+}\right]
\end{aligned}
$$

where (8) has been used and $N_{k}=\frac{I_{\ell} \beta_{k}^{2}}{\omega_{k}^{+}}$is the plasmon number. Deriving the dispersion relation from (16) is straightforward. Introducing the interaction kernels with $\beta_{q} \simeq 1$, we find, to first order in the wave energy density,

$$
\begin{aligned}
x^{2}-1 & -\sum_{k} \frac{\omega_{k}^{+}}{\beta_{k}^{4}} N_{k}+x \sum_{k} \frac{\hat{q} \cdot \frac{d N_{k}}{d \underline{\underline{k}}}}{x-\hat{q} \cdot \frac{d \omega_{k}^{+}}{d \underline{k}}} \frac{k^{4}}{\beta_{k}^{3}} \cos \theta \sin \left(k_{k}\right)+ \\
& +\sum_{k} \frac{\hat{q} \cdot \frac{d N_{k}}{d \underline{k}}}{x-\hat{q} \cdot \frac{d \omega_{k}^{+}}{d \underline{\underline{k}}}} \frac{k^{2}}{\beta_{k}^{2}}\left(\frac{k^{4}}{4 \beta_{k}^{4}}+\beta_{k}^{2} \cos ^{2} \theta\right)=0,
\end{aligned}
$$

where $x=\frac{\Omega}{q}, \underline{q}=\underline{q} / q$, and $\cos \theta=(\underline{k} \cdot q) / k_{q}$. We evaluate $\hat{q} \cdot \frac{d \omega_{k}^{+}}{d \underline{k}}$ as $\cos \theta \operatorname{sgn}\left(k_{+}\right) / \beta_{k}^{3}$.

The denominator appearing in equation (17) bears a direct analogy with that in the theory of Landau damping : the group velocity of the plasmons playing the role of the particle velocity. Taking the limit $\sum_{k} \longrightarrow \int d^{3} k$, and integrating by parts to eliminate the $\underline{k}$-derivative acting on the plasmon number, we obtain 


$$
\begin{aligned}
& x^{2}-1-\int\left|N_{k}\right| \frac{k}{\beta^{5}} d^{3} k-x^{2} \int \frac{\left|N_{k}\right|}{D_{k}^{2}}\left(3 \frac{\cos ^{2} \theta}{\beta_{k}^{2}}+1\right) \frac{k^{3}}{\beta_{k}^{3}} d^{3} k \\
& -x \int \frac{N_{k} \cos \theta}{D_{k}^{2}} \frac{k^{3}}{\beta_{k}^{6}}\left(2 \frac{\beta_{k}^{6}}{k^{2}}+\frac{3}{2} \frac{k^{2}}{\beta_{k}^{2}}-4 \cos ^{2} \theta\right) d^{3} k \\
& +\int \frac{\left|N_{k}\right|}{D_{k}^{2}} \frac{k}{\beta_{k}^{3}} \cdot\left[-\frac{k^{4}}{4 \beta_{k}^{6}}+\cos ^{2} \theta\left(\frac{3}{4} \frac{k^{4}}{\beta_{k}^{8}}+\frac{k_{k}^{4}}{\beta_{k}^{6}}+1\right)+\right. \\
& \left.+\cos { }^{4} \theta\left(1+3 \frac{k^{2}}{\beta_{k}^{2}}\right)\right] d^{3} k=0, \\
& \text { where } D_{k}=x-\operatorname{sgn}\left(k_{1}\right) \frac{\cos \theta}{\beta_{k}^{3}} .
\end{aligned}
$$

IV. Analysis

Despite all our simplifications, the dispersion relation (17) remains formidable. To solve it in full generality, one would have to use contour integrals, as in the Landau problem, since the denominator may be singular (as opposed to the case given in Ref..4). Tn this paper, however, we shall confine ourselves to instabilities of the hydrodynamic type. Furthermore, we shall make the "cold" wave approximation, i.e. we shall take a plasmon distribution of the form

$$
N_{k}=N_{0} \delta\left(\underline{k}-\underline{k}_{0}\right) \text {. }
$$


This is justified if the plasmon distribution is peaked around some k. (e.g. a shifted Gaussian) satisfying

$$
\left|D_{k_{0}}\right|>\Delta_{k}
$$

where $\Delta_{k}$ is the "thermal" spread. (19) guarantees that the regions in which $N_{k}$ is nonvanishing and $D_{k}$ vanishes are far apart, and is the validity condition of all the results that follow. One sees now why the tedious operation of integrating equation (17) by parts was carried out, since one cannot introduce directly the cold wave approximation into (17).

With this approximation, the dispersion relation (18) reduces to a quartic polynomial in $\mathbf{x}$ (normalized frequency)

$$
\begin{aligned}
& \left(x^{2}-1-|N| \frac{k}{\beta}\right)\left(x-\operatorname{sgn}\left(k_{2}\right) \cos \theta / \beta^{3}\right)^{2}-x^{2}|N| \frac{k^{3}}{\beta^{3}}\left(3 \frac{\cos ^{2} \theta}{\beta^{2}}+1\right) \\
& -x N \cos \theta \frac{k^{3}}{\beta^{6}}\left(2 \frac{\beta^{6}}{k^{2}}+\frac{3}{2} \frac{k^{2}}{\beta^{2}}-4 \cos ^{2} \theta\right) \\
& +\quad|N| \frac{k}{\beta^{3}}\left[\cos ^{4} \theta\left(1+3 \frac{k^{2}}{\beta^{2}}\right)+\cos ^{2} \theta\left(1+\frac{3}{4} \frac{k^{4}}{\beta^{2}}+\frac{k^{4}}{\beta^{6}}\right)-\frac{k^{4}}{4 \beta^{6}}\right]=0,
\end{aligned}
$$

where the subscripts have been dropped to simplify the notation.

We proceed now to analyze equation (20) for various scales of the parameters $k, \cos \theta$, and the variable $x$. 


\section{A. Perpendicular Case}

Considerable simplification occurs if the coherent wave is perpendicular to the turbulent waves, irrespective of $k$ (turbulence wavevector). The dispersion relation reduces to a biquadratic

$$
x^{4}-x^{2}\left(1+|N| \frac{k}{\beta^{5}}+|N| \frac{k^{3}}{\beta^{3}}\right)-|N| \frac{k^{5}}{4 \beta^{9}}=0 .
$$

that admits two real roots similar in form to a result obtained in Ref. 4 and two purely imaginary ones

$$
x= \pm \frac{i}{2}\left(|N| \frac{k^{5}}{\beta}\right)^{1 / 2}
$$

Then, provided

$$
|N|^{1 / 2} \gg 2 \Delta_{k}\left(\frac{\beta^{5}}{k^{5}}\right)^{1 / 2} \beta^{2}
$$

the turbulence is always unstable if the perturbation is perpendicular to the turbulent waves. Notice that the evolution due to (22) is aperiodic.*

From now on, $\cos \theta$ will be considered non-vanishing.

\footnotetext{
* A result similar to (23) has been given by Vedenov ${ }^{3}$ for the case of Langmuir turbulence.
} 


\section{B. Resonant Case}

What is meant by resonance here is situations where

$$
x=\operatorname{sgn}\left(k_{z}\right) \cos \theta / \beta^{3}+x^{(1)}
$$

with $\left|x^{\prime \prime \prime}\right| \ll|x|$, i.e. when $D_{k}$ is a small quantity... To lowest order then, (20) reduces to

$$
\begin{aligned}
& \left(1-\frac{\cos ^{2} \theta}{\beta^{6}}\right)\left(x^{\prime \prime \prime}\right)^{2}=\operatorname{IN} \frac{k}{4 \beta^{3}}\left\{4 \cos ^{4} \theta\left(1+\frac{k^{2}}{\beta^{6}}\right)\left(4-\frac{3}{\beta^{2}}\right)+\right. \\
& \left.+\cos ^{2} \theta\left[\left(\frac{5}{\beta^{2}}-\frac{3}{\beta^{4}}\right)\left(\frac{3}{\beta^{2}}-\frac{1}{\beta^{4}}\right)+4 \frac{k^{2}}{\beta^{2}}\right]-\frac{k^{4}}{\beta^{6}}\right\} .
\end{aligned}
$$

By using the properties of quadratic polynominals, we find that the turbulence is unstable if

$$
\cos ^{2} \theta<\frac{k^{2}+1}{4 k^{2}+1} .
$$

(25) is valid if

$$
|N|^{1 / 2} \gg \Delta_{k}\left(\frac{\beta^{3}}{k}\right)^{1 / 2}
$$

\section{Non-Resonant Case}

Non-resonance here refers to situations in which $D_{h}$ is of the same order as $x$, i.e.

$$
\left|x-\operatorname{sgn}\left(k_{z}\right) \cos \theta / \beta^{3}\right| \sim|x| \text {. }
$$


Factorizing (20) into the various powers of $\boldsymbol{x}$, we obtain the equivalent equation

$$
\begin{aligned}
& x^{4}-2 \operatorname{sgn}\left(k_{+}\right) \frac{\cos \theta}{\beta^{3}} x^{3}-x^{2}\left[1-\frac{\cos ^{2} \theta}{\beta^{6}}+|N| \frac{k^{3}}{\beta^{3}}\left(3 \frac{\cos ^{2} \theta}{\beta^{2}}+1\right)+|N| \frac{k}{\beta^{5}}\right] \\
& -x\left\{-2 \frac{\cos \theta}{\beta^{3}}\left[\operatorname{sgn}\left(h_{z}\right)+\frac{\sqrt{k}}{\beta^{5}}\right]+N \cos \theta \frac{k^{3}}{\beta^{6}}\left(\frac{2 \beta^{6}}{k^{2}}+\frac{3}{2} \frac{k^{2}}{\beta^{2}}-4 \cos ^{2} \theta\right)\right\} \\
& +|N| \frac{k}{\beta^{3}}\left[\cos 4 \theta\left(1+\frac{k^{2}}{\beta^{2}}\right)-\frac{k^{4}}{4 \beta^{6}}+\right. \\
& \left.+\cos ^{2} \theta\left(1+\frac{3}{4} \frac{k^{4}}{\beta^{8}}+\frac{k^{4}}{\beta^{6}}-\frac{1}{\beta^{8}}\right)\right]-\cos ^{2} \theta / \beta^{6}=0,
\end{aligned}
$$

which is more amenable to the analysis that we will now perform. We first assume that $k \gg 1$, and examine (27) for various scales of $x$.

\section{1. $x \gg 1$}

Neglecting 1 with respect to $x$, and keeping only the lowest order terms, (27) becomes

$$
x^{3}=2 N \cos \theta k
$$

which always has complex roots. The growth rate $\gamma \equiv \operatorname{Im} x$ here is given by

$$
\gamma=\frac{\sqrt{3}}{2}(2 N \cos \theta k)^{1 / 3} .
$$


(28) - (29) are valid if

$$
|N \cos \theta k|^{1 / 3} \gg \Delta_{k} \text {. }
$$

\section{2. $x \sim 1$}

We may no longer neglect 1 with respect to $\boldsymbol{x}$. We now have

$$
x^{3}-x(1+I N I)-2 N \cos \theta k=0
$$

which yields the instability condition

$$
|\cos \theta|>\frac{1}{3 \sqrt{3}|N| k}\left(1+\frac{3}{2} \mathbb{N} \mid\right)
$$

The growth rate now goes as

$$
y \sim|N \cos \theta k|
$$

since all terms in (31) are of the same order.

(31) - (33) are valid if

$$
|N \cos \theta k|>\rangle \Delta_{k} .
$$

It is worth noting that results similar in form to (29), (32) and (33) can be readily obtained from equation (18), without any assumption as to the form of the plasmon distribution, since $\operatorname{squ}\left(h_{+}\right) \frac{\cos \theta}{\beta^{3}}$ may be discarded in comparison with $x$ if $x \geqslant 1, k \gg 1$. 


\section{3. $\underline{x}<1$}

This time, $x$ will be neglected in comparison with unity. To lowest orders, equation (27) becomes a quadratic :

$$
x^{2}+2 x \cos \theta\left(N k-\frac{\operatorname{sgn}\left(k_{2}\right)}{\beta^{3}}\right)+\frac{\cos ^{2} \theta}{\beta^{6}}
$$

$$
-I N \mid \frac{k}{\beta^{3}}\left[\cos ^{4} \theta\left(1+\frac{3 k^{2}}{\beta^{2}}\right)+\cos ^{2} \theta-\frac{k^{4}}{4 \beta^{6}}\right]=0
$$

where $k^{4} / 4 \beta^{6}$ has been kept since both $\cos ^{2} \theta$ and $\cos ^{4} \theta$ may be sma11. The solutions of (35) are given by

$$
\begin{aligned}
& x=-\frac{\cos \theta}{\beta^{3}}\left[N \beta^{3}-\operatorname{sgn}\left(k_{z}\right)\right] \pm\left\{\frac{\cos ^{2} \theta}{\beta^{6}}\left[N k \beta^{3}-\operatorname{sgn}\left(k_{z}\right)\right]^{2}+\right. \\
& \left.+\quad I N \mid \frac{k}{\beta^{3}}\left[\cos ^{4} \theta\left(1+3 \frac{k^{2}}{\beta^{2}}\right)+\cos ^{2} \theta-\frac{k^{4}}{4 \beta^{6}}\right]-\frac{\cos ^{2} \theta}{\beta^{6}}\right\}^{1 / 2} .
\end{aligned}
$$

Again, using the properties of quadratic polynominals, we find the instability condition to be

$$
\cos ^{2} \theta<\frac{1}{2\left(1+\frac{3 k^{2}}{\beta^{2}}\right)}\left\{\left[\operatorname{sog}\left(k_{4}\right)-N k \beta^{3}\right]+\left[\left(|N| k \beta^{3}-1\right)^{2}+\frac{k^{4}}{\beta^{6}}\left(1+\frac{3 k^{2}}{\beta^{2}}\right)\right]^{1 / 2}\right\} \text {. }
$$

For $\left.\left.\left|N k_{\beta}^{3}\right|\right\rangle\right\rangle 1$, , (37) reduces to

$$
\cos ^{2} \theta<\frac{1}{4|N| k^{6}} .
$$


The growth rate here is quite small. It is approximately given by

$$
\gamma \sim \frac{1}{|N| k^{6}}
$$

(35) - (39) are valid if

$$
|N \cos \theta k| \gg \Delta_{k}
$$

No non-resonant instability appears for $k \leqslant 1$.

The results obtained so far apply only in limiting cases, and were sometimes obtained at the cost of drastic approximations. One is justified in questioning the accuracy of these results. Bearing this in mind, we have computed the boundaries between stable and unstable regions in the phase space $(N, k, \cos \theta)$ of the dispersion relation (20), by means of the Sturm theorem ${ }^{11}$. These boundaries are plotted in Fig, 1 and 2, along with the conditions derived analytically. We note with satisfaction that the agreement is quite good, especially for large $k$. Moreover, the two plots seem to indicate that for $\cos \theta=1$ (i.e. the one-dimensional case), only one unstable region is to be found (non-resonant, for $x \geqslant 1$ ), and that no resonant instability occurs. We have verified this numerically. It is worth mentioning that the dispersion equation derived keeping quadratic nonlinearities only gives results similar to the above only for large $k$. The reason for this is that although the cubic contribution is much smaller than the quadratic contribution for $k \gg 1$, both are of comparable magnitude for $k \leq 1$. This is due to the structure of the cubic and quadratic interaction kernels. 
V. Conclusion

We have applied a method using the correlation function together with a. Fourier-transform technique to study the interaction of weak highfrequency ion-acoustic turbulence with low-frequency ion-acousticlike perturbations.

We have found that provided certain conditions are met, the turbulence becomes unstable. These conditions have been explicitly derived and verified numerically.

It is worth noting that a one-dimensional analysis would have been incomplete, since it masks the resonant instability.

Acknowledgements

The authors are grateful to Professor E.S. Weibel for his encouragement and support and to Dr. P.D. Morgan for reading the manuscript.

This work was supported by the Swiss National Science Foundation, by Euratom, and by the Ecole Polytechnique Fédérale de Lausanne. 
References

1 H. de Kluiver, Rijnhuizen Report 77-106, FOM (1977)

2 R.Z. Sagdeev, Rev.Mod.Phys. 51, 1 (1979)

3 A.A. Vedenov, Theory of Turbulent Plasma, (Iliffe, London, 1968)

4 J. Vaclavik, K. Appert, Phys.F1uids 23, 9 (1980)

5 R.C. Davidson, Methods in Nonlinear Plasma Theory, (Academic, New York, 1972)

6 R.Z. Sagdeev, A.A. Galeev, Nonlinear Plasma Theory, (Benjamin, New York, 1969)

7 D.B. Ilic, Phys.F1uids 20, 1717 (1977)

8 Y. Kawai, M. Guyot, Phys.Rev.Lett. 39, 1141 (1977)

9 W. Gekelman, R.L. Stenzel, Phys.Fluids 21, 2014 (1978)

10 Ch. Hollenstein, M. Guyot, to be published

11 A. Kurosh, Cours d'Algèbre Supérieure (Mir, Moscow, 1973) 
Figure Captions

Fig. 1 Boundaries of the instability in the plane $\cos \theta, k$ for $N=.1$. Solid lines show the results obtained numerically. The dotted line represents the resonant instability condition (25), whereas the dotted-dashed and dashed lines represent the non-resonant instability condition $k \gg 1$, $x<<\quad$ (37), and $k \gg 1, x \sim 1 \quad$ (32), respectively.

Fig. 2 Boundaries of the instability in the plane $\cos \theta, k$ for $N=.001$. The solid line shows the results obtained numerically. The dotted line represents the resonant instability condition (25), whereas the dotted-dashed line represents the non-resonant instability condition for $k \gg 1, x<1 \quad$ (37). The non-resonant instability condition for $k \gg 1, x \sim 1$ (32) does not appear here, due to the scale of $k$. 


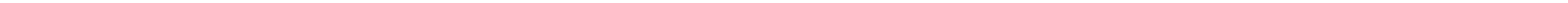

ㅁำ 


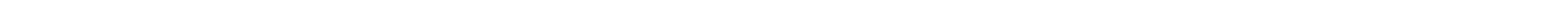

